\title{
On the Möbius transformation in the entanglement entropy of fermionic chains
}

\author{
Filiberto Ares*坾 \\ Departamento de Física Teórica, Universidad de Zaragoza, 50009 Zaragoza, Spain \\ José G. Esteve用 and Fernando Falcetd§ \\ Departamento de Física Teórica, Universidad de Zaragoza, 50009 Zaragoza, Spain and \\ Instituto de Biocomputación y Física de Sistemas Complejos (BIFI), 50009 Zaragoza, Spain \\ Amilcar R. de QueirotT \\ Departamento de Física Teórica, Universidad de Zaragoza, 50009 Zaragoza, Spain and \\ Instituto de Fisica, Universidade de Brasilia, \\ Caixa Postal 04455, 70919-970, Brasília, DF, Brazil
}

There is an intimate relation between entanglement entropy and Riemann surfaces. This fact is explicitly noticed for the case of quadratic fermionic Hamiltonians with finite range couplings. After recollecting this fact, we make a comprehensive analysis of the action of the Möbius transformations on the Riemann surface. We are then able to uncover the origin of some symmetries and dualities of the entanglement entropy already noticed recently in the literature. These results give further support for the use of entanglement entropy to analyse phase transitions.

\section{INTRODUCTION}

In the present work we make a comprehensive analysis of the symmetries and the dualities of the Rényi entanglement entropy for the ground state of quadratic, translational invariant fermionic Hamiltonians with finite range couplings in a one-dimensional chain.

\footnotetext{
* Corresponding author.

†Electronic address: ares@unizar.es

‡Electronic address: esteve@unizar.es

$\S$ Electronic address: falceto@unizar.es

๑Electronic address: amilcarq@gmail.com
} 
We here focus on the case of non-critical, that is, gapped Hamiltonians with reflection and charge conjugation symmetry.

For such Hamiltonians, the entanglement entropy associated with a partial observation on an interval of contiguous sites is a functional of the determinant of the 2-point correlation matrix. This matrix is of the block Toeplitz type [1]. It was shown [2, 3] that by using Riemann-Hilbert problem techniques one can obtain the asymptotics of this determinant. Furthermore, in the process of computing the asymptotics of the determinant one is led to cast the moduli of Hamiltonians through their dispersion relations in terms of a compact Riemann surface. The branch points of the hyperelliptic curve which defines this Riemann surface depend on the coupling constants of the Hamiltonian.

Our analysis of the symmetries and the dualities of the entanglement entropy is based on the study of the action of Möbius transformations on the above Riemann surface, which moves the branch points and, therefore, the coupling constants but leaves invariant the asymptotic expansion of the determinant of 2-point correlations and, therefore, the entanglement entropy. We then obtain the following collection of remarkable results:

1. We uncover the fact that the entanglement entropy only depends on the Möbius transformation invariants which are constructed with the branch points of the hyperelliptic curve associated with the couplings of the model. This fact explains the ellipses of constant entropy first noticed in the XY model in [4].

2. We analyse what Möbius transformations map the original couplings to other physically allowed, i.e. to other that preserve the hermiticity of the Hamiltonian. We find that these Möbius transformations are inversions and the $1+1$ Lorentz group, $S O(1,1)$. In particular, we observe that under $S O(1,1)$ the couplings change such that the dispersion relation behaves as a homogeneous field with scaling dimension equal to the maximum range $L$ of the coupling.

3. We also note that the asymptotic expansion of the determinant is also invariant under certain permutations of the branch points which induce a modular transformation of the non contractible cycles of our Riemann surface. This fact, combined with the previous invariance under Möbius transformations, can be useful to find and analyse symmetries and dualities between different Hamiltonians in terms of entanglement entropy. As an example, we apply this idea in the XY spin chain. 
In particular, the duality noticed in [4] is discussed in terms of a Dehn twist of the underlying Riemann surface which in this XY case is a torus. Recall that two distinct Dehn twists, which are two special transformations of the moduli of the torus, generate the whole modular group in that case [5]. The Dehn twists play an important role in string theory, specially in the analysis of its many dualities. See for instance [6].

4. This framework also allows to extend the relation between the entanglement entropy of two Kramers-Wannier dual Ising models and its corresponding XX model, discussed by Igloi and Juhasz in [7], to general XY models.

We organize this paper as follows. In section II we first present the most general quadratic fermionic Hamiltonian with long range couplings and reflection and charge conjugation symmetry in a chain with $N$ sites. We next obtain the corresponding dispersion relation and then write a formula for the Rényi entanglement entropy in terms of the determinant of the 2-point correlation matrix for the ground state. Following the notorious works [2, 3], we take the asymptotics of this determinant $(13)$ by constructing a compact Riemann surface from the coupling constants of the model. It is this Riemann surface that will allow us to analyse the symmetries and dualities of the entanglement entropy based on its behaviour under the Möbius transformations. In section III we make a comprehensive analysis of the Möbius transformations on the Riemann surface associated with the moduli of coupling constants. We obtain that the entanglement entropy is left invariant, which allows us to obtain entropy preserving dualities between Hamiltonians. Furthermore, the only Möbius transformations that preserve the properties of the Riemann surface imposed by the couplings are inversion and the $1+1$ Lorentz group. A beautiful outcome of this result is that under the latter the dispersion relation itself behaves as a homogeneous field with scaling dimension associated with the maximum range $L$ of the couplings. In section $[\mathrm{IV}$ we apply our general results of previous section to the XY model. We are then able to uncover the geometrical origin of some dualities already noticed in [4, 7] and to generalize them. Finally, we conclude in section $\mathrm{V}$ by summarizing our findings and discussing some prospects for the future.

This paper also contains two appendices. In appendix A, we show that the determinant of our block Toeplitz matrix is invariant under permutation of the branch points defining 
the Riemann surface. In appendix B, we recollect the important facts concerning the representation of $S L(2, \mathbb{C})$ on the space of homogeneous polynomials of two complex variables. This plays a crucial role in the understanding how the Hamiltonian, and in particular its couplings constants, change under Möbius transformations.

\section{FINITE RANGE HAMILTONIANS, COMPACT RIEMANN SURFACES AND ENTANGLEMENT ENTROPY}

Let us consider a $N$-site chain of size $\ell$ with $N$ spinless fermions described by a quadratic, translational invariant Hamiltonian with finite range couplings $(L<N / 2)$

$$
H=\frac{1}{2} \sum_{n=1}^{N} \sum_{l=-L}^{L}\left(2 A_{l} a_{n}^{\dagger} a_{n+l}+B_{l} a_{n}^{\dagger} a_{n+l}^{\dagger}-B_{l} a_{n} a_{n+l}\right)
$$

Here $a_{n}$ and $a_{n}^{\dagger}$ represent the annihilation and creation operators at the site $n$. The only non-vanishing anticommutation relations are

$$
\left\{a_{n}^{\dagger}, a_{m}\right\}=\delta_{n m}
$$

We assume periodic boundary conditions $a_{n+N}=a_{n}$ in (1).

We take $A_{l}$ and $B_{l}$ real, and in order that $H$ is Hermitian, the hopping couplings must be symmetric $A_{-l}=A_{l}$. In addition, without loss of generality, we may take $B_{l}=-B_{-l}$. For a comprehensive analysis of more general choices of couplings $A_{l}$ and $B_{l}$ see [1].

Proceeding like in [1] we introduce

$$
F_{k}=\sum_{l=-L}^{L} A_{l} e^{i \theta_{k} l}, \quad \theta_{k}=\frac{2 \pi k}{N}
$$

and

$$
G_{k}=\sum_{l=-L}^{L} B_{l} e^{i \theta_{k} l}
$$

Note that in our case, $F_{k}$ is real and $F_{-k}=F_{k}$ while $G_{k}$ is imaginary and $G_{-k}=-G_{k}$.

The Hamiltonian can now be written in diagonal form by means of anticommuting annihilation, creation operators $d_{k}, d_{k}^{\dagger}$, the Bogoliubov transform of the Fourier modes,

$$
H=\mathcal{E}+\sum_{k=0}^{N-1} \Lambda_{k}\left(d_{k}^{\dagger} d_{k}-\frac{1}{2}\right)
$$


where

$$
\mathcal{E}=\frac{1}{2} \sum_{k=0}^{N-1} F_{k}
$$

The dispersion relation reads

$$
\Lambda_{k}=\sqrt{\left(F_{k}+G_{k}\right)\left(F_{k}-G_{k}\right)}
$$

which takes non-negative values. Hence the ground state of the theory $|\mathrm{GS}\rangle$ is the vacuum of the Fock space for the $d_{k}$ modes, i.e. $d_{k}|\mathrm{GS}\rangle=0, \forall k$.

In this paper we focus our study in the Rényi entanglement entropy for the ground state of Hamiltonian (1).

Given a subset $X$ of contiguous sites of the fermionic chain, with complementary set $Y$ we have the corresponding factorization of the Hilbert space $\mathcal{H}=\mathcal{H}_{X} \otimes \mathcal{H}_{Y}$. If the system is in the pure state $|\mathrm{GS}\rangle$, the reduced density matrix for $X$ is obtained by taking the partial trace with respect to $\mathcal{H}_{Y}, \rho_{X}=\operatorname{Tr}_{Y}|\mathrm{GS}\rangle\langle\mathrm{GS}|$. The Rényi entanglement entropy of $X$ is

$$
S_{\alpha}(X)=\frac{1}{1-\alpha} \log \operatorname{Tr}\left(\rho_{X}^{\alpha}\right) .
$$

As it is well known [8 11], due to the fact that the $n$-point vacuum expectation value satisfies the Wick decomposition property, it is possible to derive the reduced density matrix from the two point correlation function. Namely, for any pair of sites $n, m \in X$, we introduce the correlation matrix

$$
\left(V_{X}\right)_{n m}=2\left\langle\left(\begin{array}{c}
a_{n} \\
a_{n}^{\dagger}
\end{array}\right)\left(a_{m}^{\dagger}, a_{m}\right)\right\rangle-\delta_{n m} I=\left(\begin{array}{cc}
\delta_{n m}-2\left\langle a_{m}^{\dagger} a_{n}\right\rangle & 2\left\langle a_{n} a_{m}\right\rangle \\
2\left\langle a_{n}^{\dagger} a_{m}^{\dagger}\right\rangle & 2\left\langle a_{n}^{\dagger} a_{m}\right\rangle-\delta_{n m}
\end{array}\right) .
$$

Following [8, 9] one can show that the Rényi entanglement entropy reads

$$
S_{\alpha}(X)=\frac{1}{2(1-\alpha)} \operatorname{Tr} \log \left[\left(\frac{\mathbb{I}+V_{X}}{2}\right)^{\alpha}+\left(\frac{\mathbb{I}-V_{X}}{2}\right)^{\alpha}\right]
$$

where $\mathbb{I}$ is the $2|X| \times 2|X|$ identity matrix and $|X|$ denotes the size of $X$.

The Cauchy's residue theorem allows us to implement (7) as

$$
S_{\alpha}(X)=\lim _{\varepsilon \rightarrow 0^{+}} \frac{1}{4 \pi i} \oint_{\mathcal{C}} f_{\alpha}(1+\varepsilon, \lambda) \frac{\mathrm{d} \log D_{X}(\lambda)}{\mathrm{d} \lambda} \mathrm{d} \lambda,
$$

where $D_{X}(\lambda)=\operatorname{det}\left(\lambda \mathbb{I}-V_{X}\right)$,

$$
f_{\alpha}(x, y)=\frac{1}{1-\alpha} \log \left[\left(\frac{x+y}{2}\right)^{\alpha}+\left(\frac{x-y}{2}\right)^{\alpha}\right]
$$




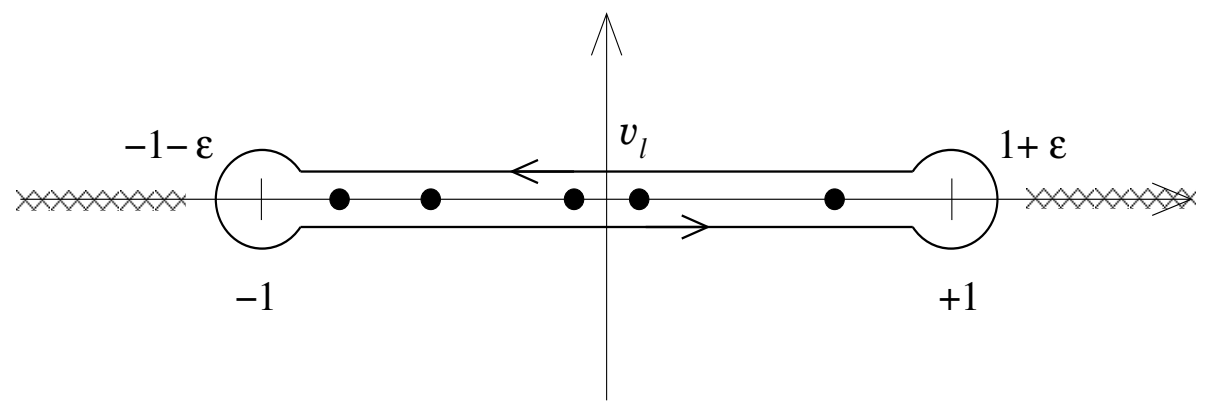

FIG. 1: Contour of integration, cuts and poles for the computation of $S_{\alpha}(X)$. The cuts for the function $f_{\alpha}$ extend to $\pm \infty$.

and $\mathcal{C}$ is the contour depicted in Fig. 1 which surrounds the eigenvalues $v_{l}$ of $V_{X}$, all of them lying in the real interval $[-1,1]$.

Applying to our case the results in [1] one can show that

$$
\left(V_{X}\right)_{n m}=\frac{1}{N} \sum_{k=0}^{N-1} M_{k} e^{i \theta_{k}(n-m)}
$$

with

$$
M_{k}=\frac{1}{\Lambda_{k}}\left(\begin{array}{cc}
F_{k} & G_{k} \\
-G_{k} & -F_{k}
\end{array}\right) .
$$

We now consider the thermodynamic limit, $N \rightarrow \infty, \ell \rightarrow \infty$ with $N / \ell$ fixed. In this limit the previous $N$-tuples, like $\Lambda_{k}=\Lambda\left(\theta_{k}\right), F_{k}=F\left(\theta_{k}\right), G_{k}=G\left(\theta_{k}\right)$ and the others, with $\theta_{k}=2 \pi k / N$, become $2 \pi$-periodic functions of the continuous variable $\theta$, that is, $\Lambda(\theta), F(\theta), G(\theta), \ldots$

In order to obtain the asymptotic behaviour of the entanglement entropy (8), we must compute $D_{X}(\lambda) \equiv \operatorname{det}\left(\lambda \mathbb{I}-V_{X}\right)$ when the size $|X|$ of $X$ is large.

This task was solved in [2, 3] by reducing it to a particular Wiener-Hopf factorization problem. To describe the result we introduce the (complex) Laurent polynomials

$$
\Theta(z)=\sum_{l=-L}^{L} A_{l} z^{l}, \quad \Xi(z)=\sum_{l=-L}^{L} B_{l} z^{l}
$$

that are related to $F$ and $G$, from $(2)$ and $(3)$, by $F(\theta)=\Theta\left(\mathrm{e}^{i \theta}\right)$ and $G(\theta)=\Xi\left(\mathrm{e}^{i \theta}\right)$. In terms of 10 we extend the symbol $M(\theta)$ to the complex plane

$$
\mathcal{M}(z)=\frac{1}{\sqrt{\Theta^{2}(z)-\Xi^{2}(z)}}\left(\begin{array}{cc}
\Theta(z) & \Xi(z) \\
-\Xi(z) & -\Theta(z)
\end{array}\right)=U\left(\begin{array}{cc}
0 & g(z) \\
g(z)^{-1} & 0
\end{array}\right) U^{-1}
$$


with

$$
U=\frac{1}{\sqrt{2}}\left(\begin{array}{cc}
1 & 1 \\
-1 & 1
\end{array}\right)
$$

and

$$
g(z)=\sqrt{\frac{\Theta(z)+\Xi(z)}{\Theta(z)-\Xi(z)}} .
$$

Now, as it is shown in [2, 3, 12, the asymptotic behaviour of the logarithmic derivative of $D_{X}(\lambda)$ verifies

$$
\begin{aligned}
\frac{\mathrm{d}}{\mathrm{d} \lambda} \ln \operatorname{det} D_{X}(\lambda) & =\frac{2 \lambda}{\lambda^{2}-1}|X|+ \\
& +\frac{1}{2 \pi} \int_{|z|=1} \operatorname{Tr}\left[\left(u_{+}^{\prime}(z) u_{+}^{-1}(z)+v_{+}^{-1}(z) v_{+}^{\prime}(z)\right) \mathcal{M}^{-1}(z)\right] \mathrm{d} z+\ldots
\end{aligned}
$$

where the prime denotes the derivative with respect to $z$ and $u_{+}, v_{+}$are the solution to the following Wiener-Hopf factorization problem:

i) $\mathcal{M}(z)=u_{+}(z) u_{-}(z)=v_{-}(z) v_{+}(z)$,

ii) with $u_{-}^{ \pm 1}(z), v_{-}^{ \pm 1}(z)$ analytic outside the unit circle and $u_{+}^{ \pm 1}(z), v_{+}^{ \pm 1}(z)$ analytic inside.

This problem has been solved in [2, 3] and the results, expressed in terms of theta functions associated to the Riemann surface determined by $g(z)$, are described in the following.

First, consider the analytic structure of $g(z)$. Actually it is a bivalued function in the complex plane, but it is a single valued meromorphic function in the Riemann surface determined by the complex curve

$$
w^{2}=P(z) \equiv z^{2 L}(\Theta(z)+\Xi(z))(\Theta(z)-\Xi(z)), \quad w, z \in \overline{\mathbb{C}}
$$

where $\overline{\mathbb{C}}$ denotes the Riemann sphere.

Here we shall assume that the polynomial $P(z)$ has $4 L$ different simple roots and therefore (12) defines a two-sheet Riemann surface of genus $\mathrm{g}=2 L-1$. On the other hand, since the real coupling constants of (1) satisfy $A_{l}=A_{-l}$ and $B_{-l}=-B_{l}$, we have

$$
\Theta\left(z^{-1}\right)=\Theta(z), \quad \overline{\Theta(z)}=\Theta(\bar{z}), \quad \Xi\left(z^{-1}\right)=-\Xi(z), \quad \overline{\Xi(z)}=\Xi(\bar{z}) .
$$

Therefore if $z_{j}$ is a root of $P(z)$ then $\bar{z}_{j}$ and $z_{j}^{-1}$ are roots as well. Actually the roots of $P(z)$ coincide with the zeros and poles of the rational function $g^{2}(z)$ and the previous 
relation specializes as follows: if $z_{j}$ is a zero (pole) of $g^{2}(z)$ then $\bar{z}_{j}$ is also a zero (pole) while $z_{j}^{-1}$ is a pole (zero).

We fix an order in the roots of $P(z)$ with the only requierement that the first half of them is inside the unit circle and the other half outside, i.e. $\left|z_{j}\right|<1, j=1, \ldots, 2 L$ and $\left|z_{j}\right|>1, j=2 L+1, \ldots, 4 L$ (notice that if all roots are simple, then necessarily $\left|z_{j}\right| \neq 1$ ).

Equipped with the previous data we fix the branch cuts of $g(z)$, which are $2 L$ non intersecting curves $\Sigma_{\rho}, \rho=0, \ldots, \mathrm{g}$ that join $z_{2 \rho+1}$ and $z_{2 \rho+2}$. Notice that it is always possible to choose them such that they do not cross the unit circle.

Associated to these cuts we have a canonical homology basis of cycles in the Riemann surface $a_{r}, b_{r}, r=1, \ldots$, g where $a_{r}$, in the upper Riemann sheet, surrounds $\Sigma_{r}$ anticlockwise and the dual cycle $b_{r}$ surrounds the branch points $z_{2}, z_{3}, \cdots, z_{2 \mathrm{~g}+1}$ clockwise. In Fig. 2 we depict a possible arrangement of the branch points and cuts for $\mathrm{g}=3(L=2)$ as well as cycles $a_{3}$ and $b_{3}$.

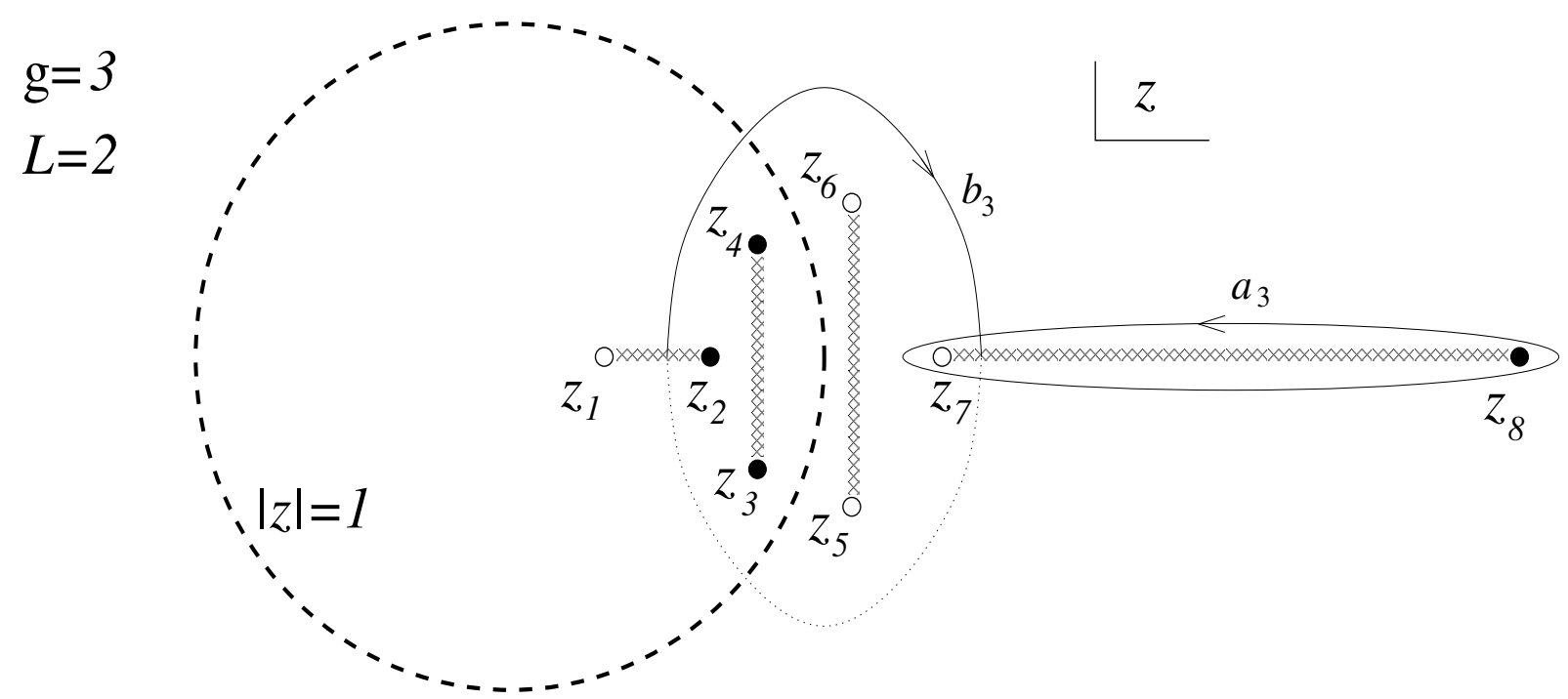

FIG. 2: Possible arrangement of the branch points and cuts of $w=\sqrt{P(z)}$ for genus $\mathrm{g}=3(L=2)$. Note that we must have $z_{1}=z_{8}^{-1}, z_{2}=z_{7}^{-1}, z_{3}=\bar{z}_{4}=\bar{z}_{5}^{-1}=z_{6}^{-1}$. The branch points in black $(\bullet)$ are zeros of $g^{2}(z)\left(\epsilon_{j}=1\right)$ while those in white (o) are poles $\left(\epsilon_{j}=-1\right)$. Oriented curves $a_{3}$ and $b_{3}$ are two of the non-contractible cycles.

The canonical basis of holomorphic forms

$$
\mathrm{d} \omega_{r}=\frac{\varphi_{r}(z)}{\sqrt{P(z)}} \mathrm{d} z,
$$


with $\varphi_{r}(z)$ a polynomial of degree smaller than $\mathrm{g}$, is chosen so that $\int_{a_{r}} \mathrm{~d} \omega_{r^{\prime}}=\delta_{r r^{\prime}}$. The $\mathrm{g} \times \mathrm{g}$ symmetric matrix of periods $\Pi=\left(\Pi_{r r^{\prime}}\right)$ is given by

$$
\Pi_{r r^{\prime}}=\int_{b_{r}} \mathrm{~d} \omega_{r^{\prime}}
$$

Associated with this matrix of periods we can now define the Riemann theta-function with characteristics $\vec{p}, \vec{q} \in \mathbb{R}^{\mathrm{g}}, \vartheta\left[\begin{array}{l}\vec{p} \\ \vec{q}\end{array}\right]: \mathbb{C}^{\mathrm{g}} \rightarrow \mathbb{C}$, given by

$$
\vartheta\left[\begin{array}{l}
\vec{p} \\
\vec{q}
\end{array}\right](\vec{s}) \equiv \vartheta\left[\begin{array}{c}
\vec{p} \\
\vec{q}
\end{array}\right](\vec{s} \mid \Pi)=\sum_{\vec{n} \in \mathbb{Z}^{\mathrm{g}}} e^{\pi i(\vec{n}+\vec{p}) \Pi \cdot(\vec{n}+\vec{p})+2 \pi i(\vec{s}+\vec{q}) \cdot(\vec{n}+\vec{p})},
$$

and its normalized version

$$
\widehat{\vartheta}\left[\begin{array}{l}
\vec{p} \\
\vec{q}
\end{array}\right](\vec{s}) \equiv \widehat{\vartheta}\left[\begin{array}{l}
\vec{p} \\
\vec{q}
\end{array}\right](\vec{s} \mid \Pi)=\frac{\vartheta\left[\begin{array}{l}
\vec{p} \\
\vec{q}
\end{array}\right](\vec{s} \mid \Pi)}{\vartheta\left[\begin{array}{l}
\vec{p} \\
\vec{q}
\end{array}\right](0 \mid \Pi)} .
$$

Finally, one has the following expression for the asymptotic value of the determinant (adapted from Proposition 1 of [3]):

$$
\log D_{X}(\lambda)=|X| \log \left(\lambda^{2}-1\right)+\log \left(\widehat{\vartheta}\left[\begin{array}{l}
\vec{\mu} \\
\vec{\nu}
\end{array}\right](\beta(\lambda) \vec{e}) \widehat{\vartheta}\left[\begin{array}{l}
\vec{\mu} \\
\vec{\nu}
\end{array}\right](-\beta(\lambda) \vec{e})\right)+\cdots,
$$

where the dots represent terms that vanish in the large $|X|$ limit. The argument of the theta function contains

$$
\beta(\lambda)=\frac{1}{2 \pi i} \log \frac{\lambda+1}{\lambda-1},
$$

and $\vec{e} \in \mathbb{Z}^{\mathrm{g}}$, a g dimensional vector whose first $L-1$ entries are 0 and the last $L$ are 1 . The characteristics of the theta function are half integer vectors $\vec{\mu}, \vec{\nu} \in(\mathbb{Z} / 2)^{\mathrm{g}}$ which are determined as follows: first assign an index $\epsilon_{j}$ to every root $z_{j}$ of $P(z)$, so that $\epsilon_{j}=1$ $\left(\epsilon_{j}=-1\right)$ if $z_{j}$ is a zero (pole) of $g^{2}(z)$; then the vectors are

$$
\begin{aligned}
\mu_{r} & =\frac{1}{4}\left(\epsilon_{2 r+1}+\epsilon_{2 r+2}\right), \\
\nu_{r} & =\frac{1}{4} \sum_{j=2}^{2 r+1} \epsilon_{j}, \quad r=1, \ldots, \mathrm{g} .
\end{aligned}
$$

Note that the indices must satisfy $\epsilon_{j}=-\epsilon_{j^{\prime}}$ whenever $z_{j}=z_{j^{\prime}}^{-1}$ and $\epsilon_{j}=\epsilon_{j^{\prime}}$ if $z_{j}=\bar{z}_{j^{\prime}}$.

In order to give an explicit expression for the determinant we have fixed an order of the roots with the only requirement that the $2 L$ first ones are inside the unit circle and the last ones outside. Of course, for consistency (13) should not depend on the chosen order. Actually it is an instructive exercise to show that the value of the determinant (in the thermodynamic limit) is in fact invariant under the transposition of two roots, provided they sit at the same side of the unit circle. In appendix $\mathrm{A}$ we outline a proof of this fact. 


\section{MÖBIUS TRANSFORMATION}

If we now perform a holomorphic bijective transformation in the Riemann sphere, $z^{\prime}=f(z)$, we move the branch points and cuts, thus modifying the holomorphic forms. But it is clear that the matrix of periods is unchanged. Therefore the theta function does not change and the entropy derived from (13) is left invariant.

It is a mathematical fact that the only holomorphic one-to-one maps of the Riemann sphere into itself are the Möbius transformations

$$
z^{\prime}=\frac{a z+b}{c z+d}, \quad\left(\begin{array}{ll}
a & b \\
c & d
\end{array}\right) \in S L(2, \mathbb{C}) .
$$

They act, see Appendix B, on the Laurent polynomial $\Theta$ by

$$
\Theta^{\prime}\left(z^{\prime}\right)=(a z+b)^{-L}\left(d z^{-1}+c\right)^{-L} \Theta(z)
$$

which is again a Laurent polynomial with monomials of degree between $L$ and $-L$. Hence, the Möbius transformation can be seen as a change of the couplings from $A_{l}$ to $A_{l}^{\prime}$. In exactly the same way we transform $\Xi$ with coefficients $B_{l}$ to a new $\Xi^{\prime}$ with coefficients $B_{l}^{\prime}$.

Notice that if we use the new Laurent polynomials to get the new rational function

$$
g^{\prime 2}=\frac{\Theta^{\prime}+\Xi^{\prime}}{\Theta^{\prime}-\Xi^{\prime}}
$$

we have $g^{\prime 2}\left(z^{\prime}\right)=g^{2}(z)$.

But this is not the end of the story because as we mentioned in the previous section, the roots of $P(z) \sqrt{12}$ satisfy certain properties, namely they come in quartets $z_{j}, \bar{z}_{j}, z_{j}^{-1}$ and $\bar{z}_{j}^{-1}$. In order to preserve this property, we must require that the Möbius transformation commute with the complex conjugation and inversion.

The commutation with conjugation restricts $S L(2, \mathbb{C})$ to the semidirect product $S L(2, \mathbb{R}) \rtimes\left\{I, i \sigma^{x}\right\}$. These are the transformations that preserve the real line. Note that the second factor in the semidirect product is related to the inversion $z^{\prime}=1 / z$ and maps the upper half plane into the lower one while the first factor contains the transformations that map the upper half plane to itself.

If we further impose that the allowed transformations must commute with the inversion, we are finally left with the group generated by the reflection $z^{\prime}=1 / z$ and the $1+1$ Lorentz 
group $S O(1,1)$ whose elements act on $z$ by

$$
z^{\prime}=\frac{z \cosh \zeta+\sinh \zeta}{z \sinh \zeta+\cosh \zeta}, \quad \zeta \in \mathbb{R}
$$

This is precisely the subgroup of the Möbius tranformations that preserve the unit circle and the real line. Its connected component maps the upper half plane and the unit disc into themselves.

We unraveled the symmetry by considering the final expression for the asymptotic determinant $(13)$, but one can trace back its origin to the Wiener-Hopf factorization problem described in the previous section. In fact, assume we perform a Möbius transformation that preserve the unit circle mapping its exterior into its exterior and the interior into the interior. Then, the property of being analytic outside (inside) the circle is preserved which implies that the solution to the Wiener-Hopf factorization for $\mathcal{M}(z)$ is transformed into that for $\mathcal{M}^{\prime}(z)$. Inserting it into (11) one immediately sees that the logarithmic derivative of $D_{X}(\lambda)$ and hence the entanglement entropy, are unchanged, at least in the asymptotic limit. Actually, a similar reasoning shows that the determinant is invariant for any Möbius transformation provided it keeps inside (outside) the unit circle the branching points that were originally inside (outside).

As for the physical interpretation of the transformations, the inversion corresponds to reverting the orientation in the chain $n \leftrightarrow N-n$ which, clearly, does not affect the entropy of the system. The coupling constants transform in a very simple way, namely, $A_{l}^{\prime}=A_{l}, B_{l}^{\prime}=-B_{l}$. The symmetries in the Lorentz group, however, have a rather non trivial implementation. In Appendix B we shall discuss in full generality how the coupling constants of the Hamiltonian behave under such Lorentz transformations.

On the other hand, it is interesting to observe that these particular Möbius transformations leave invariant not only the von Neumann entropy but also the Rényi entanglement entropy (6) for any value of $\alpha$. Actually, all the spectral properties of the two-point correlation function are preserved, at least in the large $|X|$ limit.

A very important observation is that all the previous does not apply for the critical, gapless theories, i.e. when couples of roots of $P(z)$ coincide at the unit circle. This important case will be studied separately in a future publication [13].

With respect to the dynamical aspects of these transformations, we may say that they are not a symmetry of the Hamiltonian. In the thermodynamic limit they act as a 
rescaling of the spectrum. Actually, as the unit circle is mapped into itself, we may view the transformation as a change in the momentum of the modes together with a rescaling of its energy. More concretely, adopting again the active view point we have

$$
\Lambda^{\prime}\left(\theta^{\prime}\right)=\left(\frac{\partial \theta^{\prime}}{\partial \theta}\right)^{L} \Lambda(\theta)
$$

where $\theta^{\prime}$ is the image of $\theta$ under the Möbius transformation,

$$
\mathrm{e}^{i \theta^{\prime}}=\frac{\mathrm{e}^{i \theta} \cosh \zeta+\sinh \zeta}{\mathrm{e}^{i \theta} \sinh \zeta+\cosh \zeta}
$$

and, therefore,

$$
\frac{\partial \theta^{\prime}}{\partial \theta}=\frac{1}{\sinh 2 \zeta \cos \theta+\cosh 2 \zeta} .
$$

Interestingly enough under the above transformations the dispersion relation $\Lambda(\theta)$ behaves as a homogeneous field of dimension $L$. Moreover, the dimension is directly associated with the range of the coupling. We recall that such a transformation actually corresponds to a change of the coupling constants of the theory.

The action of $S O(1,1)$ in $\overline{\mathbb{C}}$ has two fixed points in $z= \pm 1$. In particular, 1 is stable and -1 is unstable, so all the flow lines of the transformation depart from the first one and they join in the second one as we sketch in Fig. 3 .

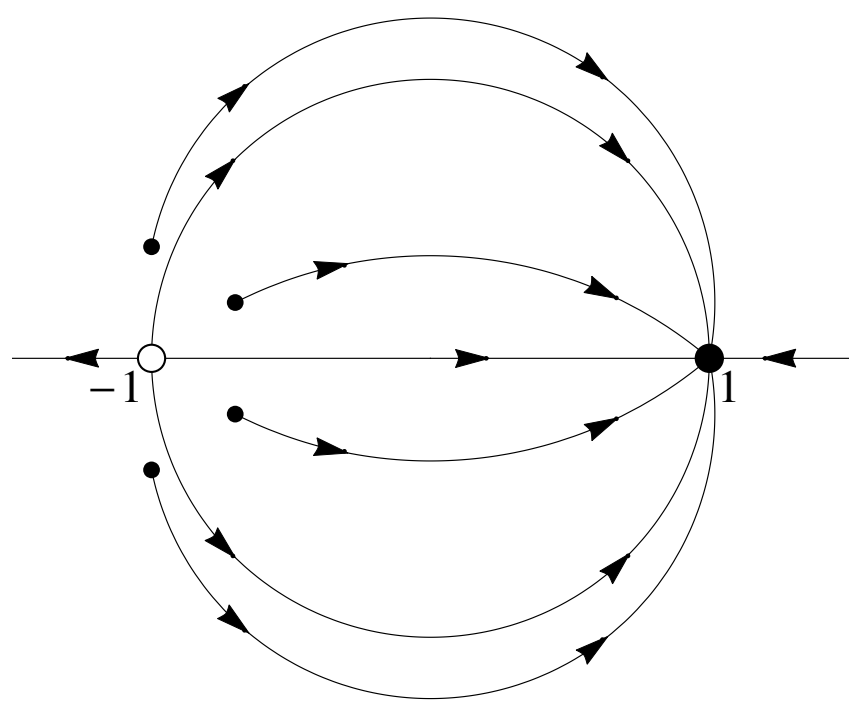

FIG. 3: Flow of $S O(1,1)$ in $\mathbb{C}$. Note that it preserves the unit circle and the real line and maps the unit disc and the upper/lower half plane into themeselves. The points $z= \pm 1$ are the fixed points of the transformation; +1 is stable and -1 unstable. 
If one considers the induced flow in the space of Hamiltonians the picture is richer. There exist $2 L+1$ fixed points whose associated complex curves are

$$
w^{2}=P(z) \equiv(z-1)^{4 L-2 j}(z+1)^{2 j}, \quad j=0, \ldots, 2 L .
$$

The only stable one corresponds to $j=0$. The other fixed points have a $j$-dimensional unstable manifold. Of course, all these Hamiltonians correspond to critical theories.

Summarizing, we have found a transformation of the coupling constants of a free fermionic chain with finite range coupling such that the spectral properties of the two-point correlation function are left invariant in the thermodynamic limit, provided the theory has a mass gap. As a byproduct we show that the asymptotic behaviour of the Rényi entanglement entropy is unchanged, whenever it is finite. On the other hand, the modes in the one particle energy spectrum are rescaled with a dimension given by the range of the couplings of the theory. The connected component of the symmetry group is composed by the Möbius transformations associated to the $1+1$ Lorentz subgroup $S O(1,1)$.

\section{THE XY SPIN CHAIN}

Our first application of the transformations introduced in the previous section is to understand the different dualities and invariances of the entanglement entropy in the XY model. This has been studied in [4]. Here we rederive their results and find some new ones using the ideas of the previous section.

The Hamiltonian reads

$$
H_{\mathrm{XY}}=\frac{1}{2} \sum_{n=1}^{N}\left[(1+\gamma) \sigma_{n}^{x} \sigma_{n+1}^{x}+(1-\gamma) \sigma_{n}^{y} \sigma_{n+1}^{y}+h \sigma_{n}^{z}\right] .
$$

The coupling constants $h$ and $\gamma$ are assumed to be real and positive.

A Jordan-Wigner transformation allows us to write this Hamiltonian in terms of fermionic operators, namely

$$
H_{\mathrm{XY}}=\sum_{n=1}^{N}\left[a_{n}^{\dagger} a_{n+1}+a_{n+1}^{\dagger} a_{n}+\gamma\left(a_{n}^{\dagger} a_{n+1}^{\dagger}-a_{n} a_{n+1}\right)+h a_{n}^{\dagger} a_{n}\right]-\frac{N h}{2},
$$

which is a particular case of the Hamiltonian of (1) with nearest neighbours coupling, $L=1$. We are now set to apply the previous results. 
The Laurent polynomials are in this case

$$
\Theta(z)=z+h+z^{-1}, \quad \Xi(z)=\gamma\left(z-z^{-1}\right)
$$

and the dispersion relation is

$$
\Lambda(\theta)=\sqrt{(h+2 \cos \theta)^{2}+4 \gamma^{2} \sin ^{2} \theta} .
$$

The theory is gapless for $h=2$ (Ising universality class) or $\gamma=0, h<2$ (XX universality class).

The zeros of $g^{2}(z)=(\Theta(z)+\Xi(z)) /(\Theta(z)-\Xi(z))$ are given by

$$
z_{ \pm}=\frac{h / 2 \pm \sqrt{(h / 2)^{2}+\gamma^{2}-1}}{1+\gamma}
$$

and its poles are the inverses $z_{ \pm}^{-1}$. Note that the critical theories correspond to the value of the couplings for which some of the zeros are in the complex unit circle. Here we do not discuss the case of critical theories, which deserves a special treatment [13]. Our focus here is on the non-critical theories, its symmetries, dualities and other properties.

If we now apply the transformations (17) the couplings of the theory change as

$$
\begin{aligned}
\gamma^{\prime} & =\frac{\gamma}{(h / 2) \sinh 2 \zeta+\cosh 2 \zeta}, \\
h^{\prime} / 2 & =\frac{(h / 2) \cosh 2 \zeta+\sinh 2 \zeta}{(h / 2) \sinh 2 \zeta+\cosh 2 \zeta},
\end{aligned}
$$

while the zeros change as

$$
z_{ \pm}^{\prime}=\frac{z_{ \pm} \cosh \zeta+\sinh \zeta}{z_{ \pm} \sinh \zeta+\cosh \zeta}
$$

and similarly for the poles.

Actually, as it was discussed in the previous section, the entropy derived from (8) and (13) is invariant under any Möbius transformation of the Riemann surface. Therefore it only depends on the zeros and poles of $g^{2}(z)$ through the Möbius invariants. For the XY model in which we have just four such points (two zeros $z_{+}, z_{-}$and two poles $z_{+}^{-1}, z_{-}^{-1}$ ) the only invariants are functions of the cross ratio

$$
x \equiv\left(z_{+}, z_{-} ; z_{+}^{-1}, z_{-}^{-1}\right)=\frac{\left(z_{+}-z_{+}^{-1}\right)\left(z_{-}-z_{-}^{-1}\right)}{\left(z_{+}-z_{-}^{-1}\right)\left(z_{-}-z_{+}^{-1}\right)} .
$$

If we use (20), this cross ratio can be written in terms of the couplings as

$$
x=\frac{1-(h / 2)^{2}}{\gamma^{2}} .
$$


The explicit form of the von Neumann entanglement entropy for non-critical XY model was computed in [2] and it was rewritten in [1, 4] in terms of the parameter $x$. See also [14, 15]. We express the von Neumann entropy in three distinct regions, namely 1a, 1b and 2 :

- Region 1a: $0<x<1$.

$$
S_{1}=\frac{1}{6}\left[\log \left(\frac{1-x}{16 \sqrt{x}}\right)+\frac{2(1+x)}{\pi} I(\sqrt{1-x}) I(\sqrt{x})\right]+\log 2 .
$$

- Region 1b: $x>1$.

$$
S_{1}=\frac{1}{6}\left[\log \left(\frac{1-x^{-1}}{16 \sqrt{x^{-1}}}\right)+\frac{2\left(1+x^{-1}\right)}{\pi} I\left(\sqrt{1-x^{-1}}\right) I\left(\sqrt{x^{-1}}\right)\right]+\log 2 .
$$

- Region 2: $x<0$.

$$
S_{1}=\frac{1}{12}\left[\log \left(16\left(2-x-x^{-1}\right)\right)+\frac{4\left(x-x^{-1}\right)}{\pi\left(2-x-x^{-1}\right)} I\left(\frac{1}{\sqrt{1-x}}\right) I\left(\frac{1}{\sqrt{1-x^{-1}}}\right)\right] .
$$

Here $I(z)$ is the complete elliptic integral of the first kind

$$
I(z)=\int_{0}^{1} \frac{\mathrm{d} y}{\sqrt{\left(1-y^{2}\right)\left(1-z^{2} y^{2}\right)}} .
$$

As it was discussed before, the fact that the entropy depends solely of the parameter $x$ can be derived as a consequence of the Möbius invariance that we uncover in this paper. A consequence of such invariance is that the Rényi entropy is a function of $x$ as well. In the following we will use the Möbius transformations to study some dualities and other relations that occur between theories in the different regions we introduce above.

\section{A. Duality between regions $1 \mathrm{a}$ and $1 \mathrm{~b}$}

Examining the expressions of the entropy in the region 1, 24) and (25), it is clear that the entropy is invariant under the change of $x$ to $x^{-1}$. We would like to understand this property in the light of the symmetries discussed before.

In fact we may derive the duality in the following way. Assume we start with a theory with couplings $\gamma_{a}>0, h_{a}$ in the region 1a, i.e., $\gamma_{a}^{2}>1-\left(h_{a} / 2\right)^{2}>0$ and $h_{a}<2$. Therefore, the points $z_{a+}$ and $z_{a-}$ are two real zeros inside the unit circle. Imagine now that we 
permute them without permuting their inverses. As it was discussed in section [II, see also Appendix A, the permutation between zeros on the same side of the unit circle does not affect the entropy, however the cross ratio 23 is now inverted: $\left(z_{a-}, z_{a+} ; z_{a+}^{-1}, z_{a-}^{-1}\right)=x^{-1}$. Note that from the point of view of the corresponding Riemann surface, which in this case is a torus, this permutation of zeros is equivalent to cut it along the $a$-cycle, perform a $2 \pi$ rotation of one of the borders, and glue them again. This is precisely one of the two Dehn twists that generate the modular group $S L(2, \mathbb{Z})$ of the torus.

One may object that the roots in the new pairs $z_{a-}, z_{a+}^{-1}$ and $z_{a+}, z_{a-}^{-1}$ are not related by inversion any more, as it should be in the XY model. Here is where the Möbius transformations come to the rescue. By suitably chosing a $S L(2, \mathbb{C})$ transformation that does not belong to $S O(1,1)$, it is posible to transform the two pairs above into $z_{b+}, z_{b+}^{-1}$ and $z_{b-}, z_{b-}^{-1}$ with the additional property $\bar{z}_{b+}=z_{b-}$. Considering now that the Möbius transformations leave the entropy invariant, we can explain the duality between (24) and 25). In particular, one may take as Möbius transformation that for which

$$
\frac{z_{b+}-z_{b-}}{1+z_{b+} z_{b-}}=-i \frac{z_{a+}-z_{a-}}{1+z_{a+} z_{a-}}
$$

These roots actually correspond to a particular choice of the couplings for the XY model, $h_{b}, \gamma_{b}$, belonging to region $1 b$, which can be related to the original ones by

$$
\gamma_{b}=\sqrt{1-\left(\frac{h_{a}}{2}\right)^{2}}, \quad \frac{h_{b}}{2}=\sqrt{1-\gamma_{a}^{2}} .
$$

In figure 4, we depict graphically in the plane $(\gamma, h)$ this choice: the point $\triangle$ has coordinates $\left(\gamma_{a}, h_{a}\right)$ and $\nabla$, with coordinates $\left(\gamma_{b}, h_{b}\right)$, is its dual.

It should be noticed that the duality, that is manifest for von Neumann also holds for the Rényi entropy (8), as it is based on the equality of the determinants $D_{X}(\lambda)$ for the two values of the couplings. On the other hand, in order to establish the duality we must perform a Möbius transformation, which implies that the dispersion relation has changed as a homogeneous field of dimension $L=1$. Therefore, in this case the spectrum of the Hamiltonian transforms non trivialy.

Before proceeding we examine for the XY model how the expressions for a general Riemann surface specializes to one with genus 1 . In this case the Riemann theta function in g complex variables reduces to the elliptic theta function with characteristics in one 
variable $\vartheta\left[\begin{array}{c}\mu \\ \nu\end{array}\right](s \mid \tau)$. The period matrix $\Pi$ is replaced by the modulus $\tau$ defined by

$$
\tau=i \frac{I(\xi)}{I\left(\sqrt{1-\xi^{2}}\right)},
$$

with

$$
\xi= \begin{cases}\sqrt{x}, & 0<x<1, \quad \text { case } 1 \mathrm{a}, \\ \sqrt{x^{-1}}, & x>1, \quad \text { case } 1 \mathrm{~b}, \\ \frac{1}{\sqrt{1-x^{-1}},} & x<0, \quad \text { case } 2,\end{cases}
$$

and the order chosen for the zeros and poles of $g^{2}(z)$ is

$$
\begin{aligned}
& z_{a+}, z_{a-}, z_{a+}^{-1}, z_{a-}^{-1}, \quad \text { for case } 1 \mathrm{a}, \\
& z_{b+}, z_{b-}, z_{b+}^{-1}, z_{b-}^{-1}, \quad \text { for case } 1 \mathrm{~b}, \\
& z_{2+}^{-1}, z_{2-}, z_{2+}, z_{2-}^{-1}, \quad \text { for case } 2,
\end{aligned}
$$

that fulfills in all cases the requirement of the previous section, i. e. the first two branch points are inside the unit circle and the last two outside. Consequently, the assignement of indices is $(+1,+1,-1,-1)$ for the cases $1 \mathrm{a}, 1 \mathrm{~b}$ and $(-1,+1,+1,-1)$ for the case 2 . Now, one can easily compute the characteristics to give $\mu_{a}=\mu_{b}=1 / 2, \nu_{a}=\nu_{b}=0$ for cases $1 \mathrm{a}, 1 \mathrm{~b}$ and $\mu_{2}=0, \nu_{2}=0$ in case 2 .

\section{B. Duality between regions 1a and 2: Kramers-Wannier duality}

We derived above a duality for the entanglement entropy that was based in the exchange of the two zeros on the same side of the unit circle. This does not change the Riemann surface and leaves the entropy invariant. Another duality that preserves the Riemann surface is the exchange of a real root with its inverse. This establishes a relation between the regions 1a and 2. This corresponds to $z_{a+}=\left(z_{2+}\right)^{-1}$. The relation between the coupling constants is then,

$$
\frac{h_{2}}{2}=\frac{2}{h_{a}} ; \quad \frac{\gamma_{2}^{2}-1}{h_{2}}=\frac{\gamma_{a}^{2}-1}{h_{a}} .
$$

In figure 4 , the point $\boldsymbol{\nabla}$ in region 2 is the dual of $\triangle$.

One immediately obtains that under this duality, the dispersion relation changes as

$$
\Lambda_{2}(\theta)=\frac{2}{h_{a}} \Lambda_{a}(\theta)
$$


which implies that up to a trivial rescaling the spectrum of the Hamiltonian is unchanged. For the particular value $\gamma_{a}=1$, in which XY reduces to the Ising model, the dual theory is also the Ising model $\left(\gamma_{2}=1\right)$ with a different magnetic field. In this case this duality coincides with the Kramers-Wannier duality [16] which, as it is notoriously known, is very useful for determining the critical point of the Ising model [17, 18].

If we now consider the behaviour of the entropy under the duality, we observe that the Riemann surface does not change and henceforth the modulus $\tau$ is invariant. However, as it was discussed above, the characteristics are transformed from $\mu_{a}=1 / 2$ to $\mu_{2}=0$ and therefore $D_{X}(\lambda)$ and the entropy are modified.

\section{Relation between the dual theories in $1 \mathrm{a}$ and 2 , with region $1 \mathrm{~b}$}

A beautiful result is that although the two dual theories in regions 1a and 2 have different entanglement entropies, they can be combined to obtain the entropy of a Hamiltonian in the region 1b. This result was first noticed in [7] for the Ising line $(\gamma=1)$. Our results extend this property to other points in the coupling space.

In order to proceed, recall the useful identity of theta functions [19] that combines the two dual theories,

$$
\widehat{\vartheta}[\underset{\nu}{\mu+1 / 2}](s \mid \tau) \widehat{\vartheta}\left[\begin{array}{l}
\mu \\
\nu
\end{array}\right](s \mid \tau)=\widehat{\vartheta}[\underset{\nu}{\mu+1 / 2}](s \mid \tau / 2) .
$$

If we apply this identity for $\mu=0, \nu=0$ to the expression (13) for the determinant we deduce that by summing up the Rényi entanglement entropy for two dual theories, like those with coupling constants $\gamma_{a}, h_{a}$ and $\gamma_{2}, h_{2}$ and modulus $\tau$, we can obtain the Rényi entropy of a third theory in the region $1 b$ with coupling constants $\gamma_{T}, h_{T}$ and modulus $\tau / 2$.

In particular, we may take

$$
h_{T} / 2=\sqrt{1-\gamma_{a}^{2}}, \quad \gamma_{T}=\gamma_{a} \frac{1-\sqrt{1-x_{a}}}{1+\sqrt{1-x_{a}}},
$$

with

$$
x_{a}=\frac{1-\left(h_{a} / 2\right)^{2}}{\gamma_{a}^{2}} .
$$

The asymptotic relation of the Rényi entropies for non critical Hamiltonians reads

$$
S_{\alpha}^{a}+S_{\alpha}^{2}=S_{\alpha}^{T},
$$


where the superindices obviously refer to the theories in the corresponding regions with the coupling constants defined above. For expample, in figure 4 , the point $\triangle$ is at $\left(\gamma_{a}, h_{a}\right)$ and $\boldsymbol{\nabla}$ at $\left(\gamma_{2}, h_{2}\right)$; then we have depicted $\bullet$ at $\left(\gamma_{T}, h_{T}\right)$, so its entanglement entropy is the sum of entanglement entropies of the two other points according to (26).

The relation is particularly simple when $\gamma_{a}=\gamma_{2}=1$ (Ising model) in which case the final theory corresponds to the XY model with zero transverse magnetic field $h_{T}=0$ and anisotropy parameter

$$
\gamma_{T}=\frac{1-h_{a} / 2}{1+h_{a} / 2} .
$$

A peculiarity of this case is that the relation (26) for the entanglement entropy holds not only in the asymptotic limit but also with finite size $|X|$, in which case it reads

$$
S_{\alpha}^{a}(|X|)+S_{\alpha}^{2}(|X|)=S_{\alpha}^{T}(2|X|)
$$

This relation has been known for some time [7] and, in particular, it has been used to derive the entanglement entropy for the critical Ising model using the known results for XX.

Another simple instance is when we consider the critical lines of the Ising and XX universality classes, $h_{a}=2$ and $\gamma_{b}=0$ respectively. In this case, we can establish a duality between the Rényi entropies of the models. If we take $h_{a}=2$ and $\gamma_{a}^{2} \leq 1$, the other two related models have coupling constants $h_{2}=2, \gamma_{2}=\gamma_{a}, \gamma_{T}=0$ and $h_{T} / 2=\sqrt{1-\gamma_{a}^{2}}$. Before writing explicitly the relation of the entanglement entropies we must consider that we are dealing with critical theories, which implies that the entropy of an interval $X$ scales logarithmically with its length $|X|$. As we did for the finite size case, in order to recover the additive relation between the entropies in the critical case, we must take different length intervals for the different theories, namely

$$
S_{\alpha}^{T}(2|X|)=2 S_{\alpha}^{a}(|X|)
$$

This is an interesting relation because, combined with the results of [20, 21] for the XX model, it allows to compute the Rényi entanglement entropy for the critical Ising universality class $\left(h_{a}=2\right)$ with $\left|\gamma_{a}\right| \leq 1$. The final result is

$$
S_{\alpha}^{a}(X)=\frac{\alpha+1}{12 \alpha} \log \frac{|X|}{\left|\gamma_{a}\right|}+\mathcal{U}_{\text {Ising }}^{\alpha}
$$


where

$$
\mathcal{U}_{\text {Ising }}^{\alpha}=\frac{\alpha+1}{6 \alpha} \log 2+\frac{1}{2 \pi i} \int_{-1}^{1} \frac{\mathrm{d} f_{\alpha}(1, \lambda)}{\mathrm{d} \lambda} \log \frac{\Gamma(1 / 2+\beta(\lambda))}{\Gamma(1 / 2-\beta(\lambda))} \mathrm{d} \lambda,
$$

with $\Gamma$ the Gamma function. We remark that the expression (27) for the entanglement entropy of the critical Ising line is a new result. Only the case $\left|\gamma_{a}\right|=1$ was previously known in the literature, see [7, 22].

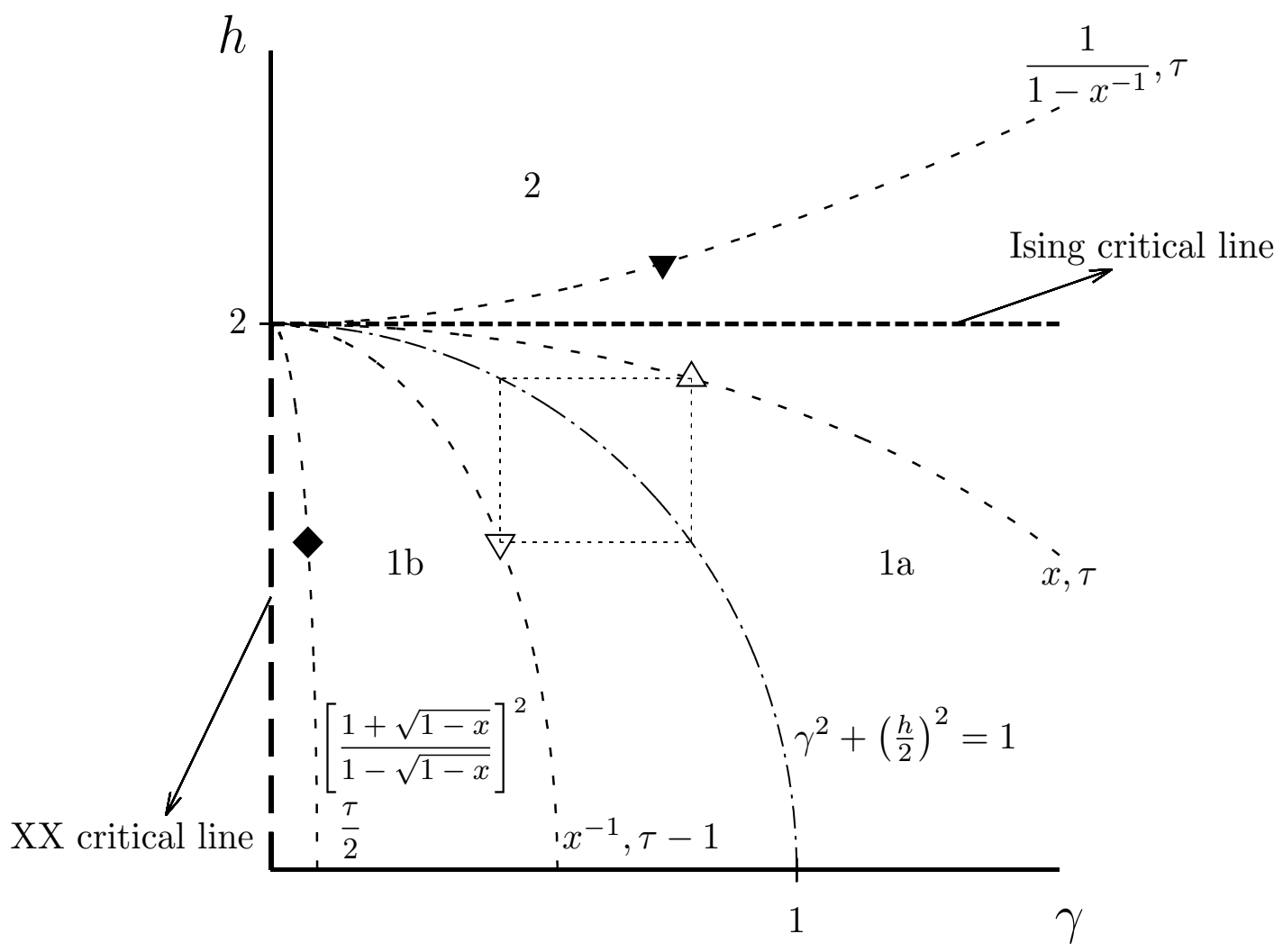

FIG. 4: Plane of couplings $(\gamma, h)$ for the XY Hamiltonian. According to the expression for the entanglement entropy, we distinguish three different regions: 1a, $1 \mathrm{~b}$ and 2. Dashed curves represent the induced flow of $S O(1,1)$. Therefore, each one connects theories with the same entanglement entropy. We also depict the different dualities and relations we have studied in the text. For each one it is written the cross ratio of the branch points and the modulus of the associated torus. The dual theory of $\Delta$ in $1 \mathrm{~b}$ is $\nabla, S_{\alpha}^{\nabla}=S_{\alpha}^{\Delta}$. The dual theory of $\Delta$ in 2 is $\boldsymbol{\nabla}$ and, although the corresponding tori have the same modulus, $S_{\alpha}^{\mathbf{v}} \neq S_{\alpha}^{\Delta}$. The entanglement entropy of the theory $\downarrow$ is given by $(26), S_{\alpha}^{\bullet}=S_{\alpha}^{\Delta}+S_{\alpha}^{\boldsymbol{\nabla}}$. 


\section{CONCLUSION}

We have seen in this work the importance of casting the space of coupling constants of a model in terms of a Riemann surface. This simple fact shows up when we are computing

the asymptotics of the determinant of the 2-point correlation matrix of the model. The next observation is that this determinant is kept invariant under Möbius transformations acting on the Riemann surface that, on the other hand, change the value of the couplings. Therefore we can have changes in the Hamiltonian that leave the determinant invariant. This fact allows us to uncover the origin and set up in a unified language the symmetries and dualities of such models.

Now, the entanglement entropy is a functional of this determinant. Therefore the many suggestions that the entanglement entropy is the right tool to study phase transitions find their explicit realization in the kind of analysis we perform in this work.

As a next step, we will extend the analysis of the Möbius transformations to the case of critical Hamiltonians [13]. This requires some other techniques since, because of the confluence of couples of branch points in the unit circle, new singularities appear.

Another direction is to extend the analysis presented in this work to the case of complex coupling constants which break reflection and charge conjugation symmetry. This case was first tackled by the authors in a recent work [1].

Acknowledgments: Research partially supported by grants 2014-E24/2, DGIID-DGA and FPA2012-35453, MINECO (Spain). FA is supported by FPI Grant No. C070/2014, DGIID-DGA. ARQ is supported by CAPES process number BEX 8713/13-8 and by CNPq under process number 305338/2012-9

\section{APPENDIX A: DETERMINANT IS INVARIANT UNDER PERMUTATIONS OF BRANCH POINTS}

In this appendix we will check the consistency of the expression for $\log D_{X}(\lambda)$ that we introduced in (13). In particular we will show that it does not depend on the order in which we choose the roots of $P(z)$, provided we take the first half inside and the last half outside of the unit circle

Suppose that we exchange the order of two roots $z_{j_{1}}$ and $z_{j_{2}}$, with $j_{\kappa}=2 r_{\kappa}+1+u_{\kappa}, \kappa=$ 
1,2 and $r_{\kappa}=1, \ldots, \mathrm{g}$ and $u_{\kappa}=0,1$. If we follow the prescriptions of section II this induces a change in the fundamental cycles which are transformed into $a_{r}^{\prime}, b_{r}^{\prime}$, such that

$$
a_{r}=\left\{\begin{array}{ll}
a_{r}^{\prime}, & r \neq r_{1}, r_{2}, \\
a_{r_{1}}^{\prime}+\Delta, & r=r_{1}, \\
a_{r_{2}}^{\prime}-\Delta, & r=r_{2}
\end{array} \quad, \quad b_{r}= \begin{cases}b_{r}^{\prime}, & r<r_{1}+u_{1}, \\
b_{r}^{\prime}+\Delta, & r_{1}+u_{1} \leq r \leq r_{2}-1+u_{2}, \\
b_{r}^{\prime}, & r>r_{2}-1+u_{2}\end{cases}\right.
$$

where

$$
\Delta=b_{r_{2}}^{\prime}-b_{r_{1}}^{\prime}+\sum_{t=r_{1}+u_{1}}^{r_{2}-1+u_{2}} a_{t}^{\prime} .
$$

This transformation is a particular instance of the most general change of basis of cycles given by a modular transform [19]

$$
\left(\begin{array}{l}
b \\
a
\end{array}\right)=\left(\begin{array}{ll}
A & B \\
C & D
\end{array}\right)\left(\begin{array}{l}
b^{\prime} \\
a^{\prime}
\end{array}\right), \quad\left(\begin{array}{ll}
A & B \\
C & D
\end{array}\right) \in S p_{2 \mathrm{~g}}(\mathbb{Z}) .
$$

The new period matrix is

$$
\Pi^{\prime}=(A-\Pi C)^{-1}(\Pi D-B) .
$$

While the normalized theta functions are related by

$$
\widehat{\vartheta}\left[\begin{array}{l}
\overrightarrow{p^{\prime}} \\
\overrightarrow{q^{\prime}}
\end{array}\right]\left(\overrightarrow{s^{\prime}} \mid \Pi^{\prime}\right)=\mathrm{e}^{-\pi i \vec{s} C \cdot \overrightarrow{s^{\prime}}} \hat{\vartheta}\left[\begin{array}{l}
\vec{p} \\
\vec{q}
\end{array}\right](\vec{s} \mid \Pi)
$$

where

$$
\overrightarrow{s^{\prime}}=\vec{s}\left(C \Pi^{\prime}+D\right)
$$

and the characteristics verify

$$
\left(\overrightarrow{p^{\prime}}, \overrightarrow{q^{\prime}}\right)\left(\begin{array}{l}
b^{\prime} \\
a^{\prime}
\end{array}\right)=(\vec{p}, \vec{q})\left(\begin{array}{l}
b \\
a
\end{array}\right)-\frac{1}{2}\left(\operatorname{diag}\left(C^{T} A\right), \operatorname{diag}\left(D^{T} B\right)\right)\left(\begin{array}{l}
b^{\prime} \\
a^{\prime}
\end{array}\right) .
$$

In the particular case of the transformation A1, after a straightforward calculation, one obtains

$$
\left(\overrightarrow{p^{\prime}}, \overrightarrow{q^{\prime}}\right)\left(\begin{array}{l}
b^{\prime} \\
a^{\prime}
\end{array}\right)=(\vec{p}, \vec{q})\left(\begin{array}{l}
b \\
a
\end{array}\right)+\frac{1}{2}\left(u_{1}+u_{2}\right)\left(b_{r_{1}}^{\prime}+b_{r_{2}}^{\prime}\right)+\frac{1}{2}\left(u_{1}+u_{2}-2\right) \sum_{t=r_{1}+u_{1}}^{r_{2}-1+u_{2}} a_{t}^{\prime} .
$$

We shall examine now how the arguments of the theta functions in $(13), \vec{s}= \pm \beta(\lambda) \vec{e}$, are modified by the transposition. Taking into account the definition of $\vec{e}$ and the form 
of the matrices $C$ and $D$ one has $\vec{e} C=0$ and $\vec{e} D=\vec{e}$ if and only if the two roots $z_{j_{1}}$ and $z_{j_{2}}$ that we exchange verify $j_{1}, j_{2} \leq 2 L$ or $j_{1}, j_{2}>2 L$, which means that both roots sit at the same side of the unit circle. In this case applying $\mathrm{A} 2$ one has $\overrightarrow{s^{\prime}}=\vec{s}= \pm \beta(\lambda) \vec{e}$.

In (15) we gave a prescription to obtain the characteristics for the theta functions involved in the computation of the entanglement entropy. They depend on the position occupied by the poles and zeros of $g^{2}(z)$ which are labeled by a sign $\epsilon_{j}$. If we exchange two of them, the original characteristics $\vec{\mu}, \vec{\nu}$ change into

$$
\tilde{\mu}_{r}=\left\{\begin{array}{ll}
\mu_{r}, & r \neq r_{1}, r_{2}, \\
\mu_{r_{1}}+\frac{1}{4} \delta, & r=r_{1}, \\
\mu_{r_{2}-\frac{1}{4} \delta,} & r=r_{2},
\end{array} \quad \tilde{\nu}_{r}= \begin{cases}\nu_{r}, & r<r_{1}+u_{1}, \\
\nu_{r}+\frac{1}{4} \delta, & r_{1}+u_{1} \leq r \leq r_{2}-1+u_{2}, \\
\nu_{r}, & r>r_{2}-1+u_{2},\end{cases}\right.
$$

where $\delta=\epsilon_{j_{2}}-\epsilon_{j_{1}}$. These, in general, are different from those obtained by the application of A3 to $\vec{\mu}$ and $\vec{\nu}$ which we denote by $\overrightarrow{\mu^{\prime}}$ and $\overrightarrow{\nu^{\prime}}$. After a somehow lengthy but direct computation one obtains

$$
\begin{aligned}
\left(\overrightarrow{\tilde{\mu}}-\overrightarrow{\mu^{\prime}}, \overrightarrow{\tilde{\nu}}-\overrightarrow{\nu^{\prime}}\right)\left(\begin{array}{c}
b^{\prime} \\
a^{\prime}
\end{array}\right)= & \left(u_{1} \frac{\epsilon_{j_{1}}-1}{2}-u_{2} \frac{\epsilon_{j_{2}}+1}{2}\right) b_{r_{2}}^{\prime}-\left(u_{1} \frac{\epsilon_{j_{1}}+1}{2}-u_{2} \frac{\epsilon_{j_{2}}-1}{2}\right) b_{r_{1}}^{\prime} \\
& +\left(\left(1-u_{1}\right) \frac{\epsilon_{j_{1}}-1}{2}-\left(1-u_{2}\right) \frac{\epsilon_{j_{2}}+1}{2}\right) \sum_{t=r_{1}+u_{1}}^{r_{2}-1+u_{2}} a_{t}^{\prime} .
\end{aligned}
$$

The important point to notice here is that, given that $u_{\kappa}=0,1$ and $\epsilon_{j}= \pm 1$, one always has $\overrightarrow{\tilde{\mu}}-\overrightarrow{\mu^{\prime}}, \overrightarrow{\tilde{\nu}}-\overrightarrow{\nu^{\prime}} \in \mathbb{Z}^{\mathrm{g}}$. This implies that

$$
\widehat{\vartheta}\left[\begin{array}{l}
\vec{\mu} \\
\vec{\nu}
\end{array}\right]=\widehat{\vartheta}\left[\begin{array}{l}
\overrightarrow{\mu^{\prime}} \\
\overrightarrow{\nu^{\prime}}
\end{array}\right],
$$

as one can easily check from the definitions.

Finally, putting everything together one has

$$
\widehat{\vartheta}\left[\begin{array}{c}
\vec{\mu} \\
\overrightarrow{\tilde{\nu}}
\end{array}\right]\left( \pm \beta(\lambda) \vec{e} \mid \Pi^{\prime}\right)=\widehat{\vartheta}\left[\begin{array}{l}
\overrightarrow{\mu^{\prime}} \\
\overrightarrow{\nu^{\prime}}
\end{array}\right]\left( \pm \beta(\lambda) \vec{e} \mid \Pi^{\prime}\right)=\widehat{\vartheta}\left[\begin{array}{l}
\vec{\mu} \\
\vec{\nu}
\end{array}\right]( \pm \beta(\lambda) \vec{e} \mid \Pi),
$$

where for the second equality we assume that the two branch points, whose order was exchanged, belong both to the first half of the ordering $\left(j_{1}, j_{2} \leq 2 L\right)$ or both to the second half $\left(j_{1}, j_{2}>2 L\right)$.

From the equality of the normalized theta-functions we deduce that a change in the order in which we take the roots does not affect the expression for the determinant, provided we do not exchange a root inside the unit circle with one outside. 


\section{APPENDIX B: MÖBIUS TRANSFORMATIONS ON THE SPACE OF HOMOGENEOUS POLYNOMIALS}

In order to obtain how Laurent polynomials (10) and, accordingly, our Hamiltonian (1) change under Möbius transformations we can study the representations of $S L(2, \mathbb{C})$ on the space of homogeneous polynomials of two complex variables [23]. In general, adopting the passive point of view, to each element $V=\left(\begin{array}{ll}a & b \\ c & d\end{array}\right) \in S L(2, \mathbb{C})$ corresponds the linear transformation in $\mathbb{C}^{2}$

$$
\left(z_{1}, z_{2}\right) \mapsto\left(d z_{1}-b z_{2},-c z_{1}+a z_{2}\right)
$$

Associated with this transformation we have the operator $T_{V}$ which acts on the space of functions $f: \mathbb{C}^{2} \rightarrow \mathbb{C}$, such as

$$
T_{V} f\left(z_{1}, z_{2}\right)=f\left(d z_{1}-b z_{2},-c z_{1}+a z_{2}\right)
$$

Note that $T_{V}$ is a reducible representation of $S L(2, \mathbb{C})$ in the space of functions of two complex variables because this space contains an invariant subspace under $T_{V}$ : the space $\mathfrak{H}_{2 L}$ of homogeneous, two variable polynomials of degree $2 L$,

$$
h\left(z_{1}, z_{2}\right)=\sum_{j=-L}^{L} u_{j} z_{1}^{L+j} z_{2}^{L-j}
$$

The restriction of $T_{V}$ to $\mathfrak{H}_{2 L}, T_{V}^{\mathfrak{H}}$, is an irreducible representation of $S L(2, \mathbb{C})$ on the space of two complex variable functions.

We can realize the space of Laurent polynomials $\mathfrak{L}_{L}$ of degree $L$ in one (complex) variable from $\mathfrak{H}_{2 L}$. In fact, any Laurent polynomial

$$
\Upsilon(z)=\sum_{l=-L}^{L} u_{l} z^{l}
$$

can be expressed like

$$
\Upsilon(z)=z^{-L} h(z, 1)
$$

Therefore, taking into account the homogeinity of $h\left(z_{1}, z_{2}\right)$, we arrive at the representation of $S L(2, \mathbb{C})$ in the space of Laurent polynomials

$$
T_{V}^{\mathfrak{L}} \Upsilon(z)=(d z-b)^{L}\left(-c+a z^{-1}\right)^{L} \Upsilon\left(\frac{d z-b}{-c z+a}\right)
$$


which is just the passive point of view of expression (16).

Now let us choose as a basis of $\mathfrak{L}_{L}$ the monomials $\left\{z^{m}\right\}$ with $-L \leq m \leq L$, and the $S U(2)$-invariant scalar product for which

$$
\left(z^{m}, z^{n}\right)=\delta_{m n}
$$

The matrix elements of $T_{V}^{\mathfrak{L}}$ in this basis are

$$
t_{m n}^{(V)}=\left(z^{m}, T_{V}^{\mathfrak{L}} z^{n}\right)
$$

After a bit of algebra and using (B1), (B2) and Newton's binomial theorem we arrive to

$$
\begin{aligned}
& t_{m n}^{(V)}=[(L+m) !(L-m) !(L+n) !(L-n) !]^{-1 / 2} \\
& \sum_{j=\max (0, m-n)}^{\min (L-n, L+m)}\left(\begin{array}{c}
L-n \\
j
\end{array}\right)\left(\begin{array}{c}
L+n \\
L+m-j
\end{array}\right)(-1)^{n-m} a^{L-n-j} b^{j} c^{n-m+j} d^{L+m-j} .
\end{aligned}
$$

Therefore the coefficients of the Laurent polynomial $\Upsilon(z)$ transform under $V \in$ $S L(2, \mathbb{C})$ as follows

$$
u_{l}^{\prime}=\sum_{m=-L}^{L} t_{l m}^{(V)} u_{m} .
$$

Since the coefficients of our Laurent polynomials $\Theta(z)$ and $\Xi(z)$ are precisely the couplings of our Hamiltonian (1), we have just found their behaviour under Möbius transformations,

$$
A_{l}^{\prime}=\sum_{m=-L}^{L} t_{l m}^{(V)} A_{m} ; \quad B_{l}^{\prime}=\sum_{m=-L}^{L} t_{l m}^{(V)} B_{m} .
$$

Note that $t_{m, n}^{(V)}=t_{-m,-n}^{(V)}$ when $a=d$ and $b=c$, as it happens for $S O(1,1)$. Therefore, in that case, the new couplings satisfy the requiered properties $A_{-l}=A_{l}$ and $B_{-l}=-B_{l}$.

[1] F. Ares, J. G. Esteve, F. Falceto, A. R. de Queiroz, Entanglement in fermionic chains with finite range coupling and broken symmetries, Phys. Rev. A 92, 042334 (2015), arXiv: 1506.06665 [quant-ph]

[2] A. R. Its, B. Q. Jin, V. E. Korepin, Entropy of XY spin chain and block Toeplitz determinants, Fields Institute Communications, Universality and Renormalization, vol 50, page 151, 2007, arXiv: quant-ph/06066178v3 
[3] A. R. Its, F. Mezzadri, M. Y. Mo, Entanglement entropy in quantum spin chains with finite range interaction, Comm. Math. Phys. Vo. 284 117-185 (2008), arXiv: 0708.0161v2 [math-ph]

[4] F. Franchini, A. R. Its, B.-Q. Jin, V. E. Korepin, Ellipses of Constant Entropy in the XY Spin Chain J. Phys. A: Math. Theor. 40 (2007) 8467-8478 arXiv: quant-ph/0609098

[5] L. Alvarez-Gaume, G. W. Moore and C. Vafa, Theta Functions, Modular Invariance and Strings, Commun. Math. Phys. 106 (1986) 1.

[6] R. Blumenhagen, D. Lst and S. Theisen, Basic concepts of string theory, Springer Science \& Business Media, 2012.

[7] F. Iglói, R. Juhász, Exact relationship between the entanglement entropies of XY and quantum Ising chains, Europhys. Lett. 81, 57003 (2008), arXiv: 0709.3927 [cond-mat.stat-mech]

[8] G. Vidal, J.I. Latorre, E. Rico, A. Kitaev, Entanglement in quantum critical phenomena, Phys. Rev. Lett. 90, 22: 227902-227906 (2003); arXiv:quant-ph/0211074v1

[9] I. Peschel, Calculation of reduced density matrices from correlation functions, J. Phys. A: Math. Gen. 36, L205 (2003); arXiv:cond-mat/0212631v1 (2002)

[10] A. P. Balachandran, T. R. Govindarajan, A. R. de Queiroz and A. F. Reyes-Lega, Entanglement and Particle Identity: A Unifying Approach Phys. Rev. Lett. 110080503 (2013) arXiv:1303.0688 [hep-th], arXiv:1205.2882 [quant-ph].

[11] A. P. Balachandran, T. R. Govindarajan, A. R. de Queiroz and A. F. Reyes-Lega, Algebraic Approach to Entanglement and Entropy Phys. Rev. A 88022301 (2013) arXiv:1301.1300 [math-ph]

[12] H. Widom, Asymptotic Behavior of Block Toeplitz Matrices and Determinants, Adv. Math. 13, 284-322 (1974)

[13] F. Ares, J. G. Esteve, F. Falceto, A. R. de Queiroz, in preparation.

[14] F. Franchini, A. R. Its, V. E. Korepin, Rényi entropy of the XY spin chain, J. Phys. A: Math. Theor. 41 (2008) 025302, arXiv: 0707.2534v4 [quant-ph]

[15] I. Peschel, On the entanglement entropy for an XY spin chain, J. Stat. Mech. (2004) P12005 arXiv: cond-mat/0410416

[16] Z. Kádar, Z. Zimborás, Entanglement entropy in quantum spin chains with broken reflection symmetry, Phys. Rev. A 82, 032334 (2010), arXiv: 1004.3112v4 [quant-ph]

[17] H. A. Kramers and G. H. Wannier, Statistics of the two-dimensional ferromagnet. Part 1., 
Phys. Rev. 60 (1941) 252.

[18] H. A. Kramers and G. H. Wannier, Statistics of the Two-Dimensional Ferromagnet. Part II, Phys. Rev. 60 (1941) 263.

[19] J. Igusa, Theta Functions, Die Grundlehren der mathematischen Wissenschaften 194, Springer-Verlag (1972)

[20] B.-Q. Jin, V.E. Korepin Quantum Spin Chain, Toeplitz Determinants and Fisher-Hartwig Conjecture J. Stat. Phys. 116 (2004) 157-190, arXiv: quant-ph/0304108

[21] F. Ares, J. G. Esteve, F. Falceto, E. Sánchez-Burillo Excited state entanglement in homogeneous fermionic chains, J. Phys. A: Math. Theor. 47 (2014) 245301 arXiv: 1401.5922 [quant-ph]

[22] J. Cardy, O. Castro-Alvaredo, and B. Doyon, Form factors of branch-point twist fields in quantum integrable models and entanglement entropy J. Stat. Phys. 130, 129 (2008) arXiv: 0706.3384 [hep-th]

[23] N. Ja. Vilenkin, Special functions and the theory of group representations, Translations of Mathematical Monographs, 22, AMS (1968) 